\title{
Short- and long-term retention of affectively varied modeled behavior'
}

\author{
DAVID J. HICKS, DEPARTMENT OF PSYCHOLOGY, CHICO \\ STATE COLLEGE, Chico, Calif. 95926
}

Children rated the affective meaning of various modeled behaviors and subsequently recalled them after two months and eight months. Short-term retention was significantly greater for positively valenced stimuli, but no difference was found on the long-term measure. Intertrial retention was interpreted as a function of subjective organizational factors.

Previous research has shown that $S$ s tend to recall attitudeconsonant material more readily than that which is attitudediscrepant and that such differences in retrieval remain up to five weeks (Levine \& Murphy, 1943). However, recent research on imitative learning has demonstrated that children will retain considerable amounts of presumedly dissonant film-mediated stimuli (physical aggression) up to six months after exposure (Hicks, 1965). Therefore, the present investigation was aimed at determining the influence of children's attitudes toward certain film-mediated behaviors on their incentive-induced short- and long-term memory of these events.

\section{METHOD}

The Ss were 21 laboratory school girls ranging in age from 7 to 9 years. Each $S$ was individually exposed to a film-mediated, female peer model performing 24 different behaviors, each for approximately $15 \mathrm{sec}$. Following each behavioral display the Ss rated each behavior on a five-point evaluative scale consisting of the following labels (with corresponding numerical values): "awful" (1), "kind of awful" (2), "not awful, not nice" (3), "kind of nice" (4), and "nice" (5). Procedures were followed to convince the children that their evaluations would remain anonymous although each child's ratings were coded and recorded as soon as the child left the experimental room.

The experimental series of modeling behaviors consisted of only 16 of 24 behaviors displayed. The additional eight units of behavior showed the model performing nonmanipulative physical activities (e.g., hopping, running, turning somersaults). These behaviors were inserted to partially disguise the correspondence between the film used to obtain attitudes and the pre-retention test film used later in the experiment. The eight behaviors were placed at the beginning and after every two behaviors of the experimental series. The behaviors in the experimental series were shown in the following order: punching an inflated plastic clown; drawing on a blackboard with chalk; kicking a large stuffed dog; bowling a ball into pins; hitting the inflated clown with a bat; throwing dishes at a target; punching the stuffed dog; throwing balls at a target; dressing a male doll; shooting darts at a target: kicking the inflated doll; playing with an electric road race set; hitting the stuffed dog with a bat; dressing a female doll; shooting darts at the inflated doll; and throwing balls at the stuffed dog.

Short-term retention measure. Two months after the ratings had been obtained a different $E$ individually exposed the children to a film showing the same model performing the 16 experimental behaviors in an uninterrupted series. Following the film and a 15-min period in which the children had an opportunity to perform the modeled behaviors each $S$ was taken to a different room where the initial measure of retention was obtained. Each child was informed that the person remembering the most behaviors from the immediately preceding film would receive a brightly wrapped, though unidentified, prize. This procedure was cnlisted to maximize efforts at recall and minimize the S's subsecjuent discussion of the experimental procedure with other children. This latter purpose was emphasized by the E telling the children that any advance knowledge of the tusk would assist other $S s$ in achieving high retention scores. Each child described the belaviors and if the $\mathrm{E}$ was unsure if the verbal description was an adequate representation of a modeled event the child was asked to demonstrate the behavior motorically. The $\mathrm{E}$ recorded both the number and order of modeled stimuli recalled. The number of accurate verbal descriptions and/or performance demonstrations made by the children were utilized as the measure of their retention for film-mediated events.

Long-term retention measure. Eight months after the initial retention scores had been obtained the children were individually retested following an identical procedure with that used for short-term retention with the single exception that the modeling film and performance opportunity were not provided. The purpose given the children for the second test was that a considerable number of tie scores necessitated a repeat scoring in order to determine the prize winner. The E conducting the two retention tests had no knowledge of the children's attitudes toward the behaviors.

\section{RESULTS AND DISCUSSION}

Short-term retention. The percentage of modeled behaviors recalled during the first retention test corresponding to attitude ratings of $1,2,3,4$, and 5 were: $61.68 \%, 63.33 \%, 78.13 \%$, $78.49 \%$, and $79.49 \%$, respectively. In order to determine whether the sign of a child's attitude toward a behavior produced differences in short-term retention the proportion of behaviors rated 1 and 2 by a child which were retained during the first retention test (e.g., the number of behaviors rated 1 and 2 which were retained over the number of behaviors rated 1 and 2) were compared with the proportion of behaviors rated 4 and 5 by the same child which were retained. Four children who did not rate any behaviors 1 or 2 could not be utilized in this or similar subsequent comparisons. Each such pair of proportions was transformed into angles. An analysis of differences between these transformed scores proved highly significant $(t=3.47$, $\mathrm{df}=16$, $\mathrm{p}<.01$ ) indicating that positively valenced behaviors are recalled more readily than negatively valenced behaviors shortly after exposure to film-mediated stimuli. Such short-term retention differences may primarily reflect disparities in the girls' acquisition of affectively varied modeling stimuli. Factors in the Ss' learning histories, motivational variables. or expectancies concerning possible post-exposure treatment could have served to selectively increase or reduce the children's attention to or perception of modeling stimuli.

Long-term retention. After eight months the percentage of originally displayed behaviors still retained for the ratings of 1,2 , 3,4 and 5 were: $35.51 \%, 43.33 \%, 48.43 \%, 37.88 \%$, and $41.03 \%$, respectively. The influence of attitudes on long-term retention was assessed by calculating and comparing, in the same manner previously described, the proportions of negatively vs positively valenced behaviors (e.g., ratings of 1 and 2 vs 4 and 5) recalled eight months after exposure. This comparison revealed that no significant difference in retention of film-mediated belavior remains over an extended interval as a function of the behavior's affective valence $(t<1)$. While differences in acquisition owing to selective perception may account for the results of the short-term retention measure, such an explanation appears unsuitable in accounting for the failure of children's attitudes toward modeling stimuli to serve as a discriminative variable in their recall after a prolonged period of time.

In an attempt to clarify the basis for this finding the proportions of positively rated behaviors recalled on the shortterm measure were compared with the proportions of the positively rated behaviors recalled after eight months. The same analysis was performed for the negatively rated behaviors. These comparisons show that the recall of positively valenced behaviors decreased significantly between trials $(t=2.40, \mathrm{dt}=16, \mathrm{p}<.05$. while the decrease in retention for negatively valenced events was not significant $(t=1.35)$. According to the findings of Tulving 
(1964) intertrial retention is a function of the individual's ability to locate or impose an organization on the material presented. Thus, it is possible that some disproportionate presence of unintended, nonaffective organizational factors, rather than attitudinal variables, could be responsible for the uneven decline in retention for affectively positive and negative modeling events.

Inspection of the 16 behaviors comprising the modeling series revealed that two obvious organizational factors (motoric forms and objects to which behavior was directed) did seem to have been present. Only three of the 16 behaviors presented to the children (bowling, drawing, and road racing) did not seem to possess these obviously relational features with another behavior in the series. Of the remaining 13 behaviors six had mean attitude ratings below 2.00 (the negative criterion) while none had a mean rating above 4.00 (the positive criterion). Therefore, it would appear that more organization-facilitated retrieval was possible for negatively valenced behaviors. A count of the number of paired recalls (clusters) involving identical motoric forms or objects to which behavior was directed was performed according to procedures for locating subjective organizational factors described by Cofer (1965). Inspection of the order of recall for all children showed that 22 clusters involving attitude-discrepant stimuli (ratings of 1 or 2) were present during long-term recall while only 14 clusters involving attitude-consonant behaviors (ratings of 4 or 5 ) could be located. The disproportionate presence of organizational factors possibly favored and stabilized the long-term reconstruction or retrieval of negatively valenced behaviors while the relative difficulty in organizing positively valenced behaviors may have been responsible for the significant, and perhaps normal, decrease in memory for these events.

Additional research incorporating a counterbalancing of affective valence and organizational factors appears necessary in order to appropriately determine the contribution of attitudes toward film-mediated modeling events in retention of these events. Nevertheless, the present results do demonstrate that children will retain the majority of their initial learning of film-mediated behaviors over an extended period of time, even those portions of modeling displays that may be personally objectionable. This general finding would seem to be of special significance in light of the wide range of behaviors currently modeled in the audio-visual form of mass media and the frequency of children's exposure to this form of presentation.

\section{REFERENCES}

COFER, C. N. On some factors in the organizational characteristics of free recall. Amer. Psychologist, 1965, 20, 261-272.

HICKS, D. J. Imitation and retention of film-mediated aggressive peer and adult models. J. Pers. soc. Psychol., 1965, 2, 97-100.

LEVINE, J. M., \& MURPHY, G. The learning and forgetting of controversial material. J. abnorm soc. Psychol., 1943, 38, 507-517.

TULVING, E. Intratrial and intertrial retention: Notes toward a theory of free recall verbal learning. Psychol Rev., 1964, 71, 219-273.

\section{NOTE}

1. This research was supported in part by Research Grant MH-10189 from the National Institutes of Health, U.S. Public Health Service. 